\title{
Target Prediction and Molecular Mechanism of Compound Honggencao for Upper Respiratory Tract Infection Based on Network Pharmacology
}

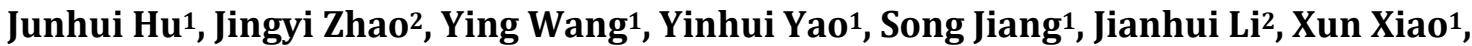 \\ Yanping Jiang1, Yanwu Zhao ${ }^{{ }^{*}}$
}

${ }^{1}$ Department of Pharmacy, The Affiliated Hospital of Chengde Medical College, Chengde, China

${ }^{2}$ Students Office, Chengde Medical College, Chengde, China

Email: *cyfyzyw@163.com

How to cite this paper: Hu, J.H., Zhao, J.Y., Wang, Y., Yao, Y.H., Jiang, S., Li, J.H., Xiao, X., Jiang, Y.P. and Zhao, Y.W. (2020) Target Prediction and Molecular Mechanism of Compound Honggencao for Upper Respiratory Tract Infection Based on Network Pharmacology. Chinese Medicine, 11, 19-29.

https://doi.org/10.4236/cm.2020.111003

Received: February 4, 2020

Accepted: March 28, 2020

Published: March 31, 2020

Copyright $\odot 2020$ by author(s) and Scientific Research Publishing Inc. This work is licensed under the Creative Commons Attribution International License (CC BY 4.0).

http://creativecommons.org/licenses/by/4.0/

\begin{abstract}
Objective: The target prediction and molecular mechanism of compound Honggencao in the treatment of upper respiratory tract infection were investigated based on network pharmacology. Methods: In the database of Traditional Chinese Medicine Systems Pharmacology Database and Analysis Platform, chemical composition and potential targets of compound Honggencao were mined, and the target gene of upper respiratory tract infection of compound Honggencao was extracted from GeneCards databases. The protein-protein interaction of target genes was constructed. Then, the essential genes of enrichment of KEGG pathway analysis and functional analysis were analysed. Results: Compound Honggencao had 69 kinds of active ingredients. The upper respiratory tract infection of the target gene was 186 that built compound Honggencao on the relationship between upper respiratory tract infections of protein interaction networks, which had a total of 186 nodes, 3515 sides. Fifty-six essential genes were including IL-17, EGFR and CDND1, and so on. Gene ontology analysis had 2567 items, and pathway analysis was 166 items. The main signaling pathways involved with IL-17 signaling pathways, tumour necrosis factor signal pathway and human cytomegalovirus infection, and so on. Conclusion: The pharmacological action of compound Honggencao on upper respiratory tract infection was characterized by the synergistic effect of multiple components and multiple targets, which provided an absolute theoretical basis for the research on the pharmacological direction of molecular signaling pathway and a specific theoretical basis for clinical use.
\end{abstract}




\section{Keywords}

Network Pharmacology, Compound Honggencao, Upper Respiratory Tract Infection, Target, Molecular Mechanism

\section{Background}

Upper respiratory tract infection is to point to the generic name of acute inflammation of nasal cavity, pharynx and larynx, and its pathogen includes virus, a bacterium, which is often used antibacterial drugs in clinical. But the use of antibiotics in clinical applications has many problems, allergic reactions and drug toxicity. Extensive use of antimicrobial agents can lead to the emergence of resistant bacteria. Serious adverse reactions such as dysbacteriosis, bring new problems to the patient's clinical treatment and rehabilitation. Therefore, to avoid the abuse of antibiotics in clinical treatment, there is often a tendency to combine Chinese patent medicine and western medicine, so reasonable combination can not only improve the drug efficacy but also reduce the bacterial resistance [1] [2]. As a conventional Chinese patent medicine in clinical practice, compound Honggencao is mainly used as Honggencao, Jinyinhua, Chuanxinlian, Yuxingcao and Yejuhua, which has the function of clearing heat and detoxifying, relieving pharynx carbuncle [3] [4]. In clinical practice, compound Honggencao is mainly used for indications such as acute pharyngitis, tonsillitis and enteritis, while the molecular mechanism of upper respiratory tract infection for acute pharyngitis is still unclear and has not been reported. Therefore, this paper will discuss the target prediction and molecular mechanism of network pharmacology in the treatment of upper respiratory tract infection, to provide some theoretical basis for clinical use.

\section{Materials and Methods}

\subsection{Collection and Screening of Chemical Components of Traditional Chinese Medicine}

Traditional Chinese Medicine Systems Pharmacology Database and Analysis Platform (TCMSP) was used to retrieve compound Honggencao, which mainly consists of five parts: Honggencao, Jinyinhua, Chuanxinlian, Yuxingcao and Yejuhua [5] [6]. The main chemical components of compound Honggencao were screened according to the ADME principle: oral bioavailability $(\mathrm{OB}) \geq$ $30 \%$, class drug resistance $(\mathrm{DL}) \geq 0.18$, and the corresponding targets of the main chemical components were obtained.

\subsection{Construction of the Compound-Target Network}

Search and download candidate genes about "upper respiratory tract infection" from the GeneCards database (www.genecards.org). The above candidate genes were compared with the target genes of the main chemical components of com- 
pound Honggencao, to select the common target genes. The software Cytoscape 3.6.1 was used to visualise the network diagram of the main chemical components of compound Honggencao-target genes of upper respiratory tract infection.

\subsection{Protein Interaction Network Construction}

Compound Honggencao main chemical composition corresponds to the upper respiratory tract infection target Genes through the STRING (Search Tool for the Retrieval of Interacting Genes/Proteins, http://sting-db.org) construction of protein interaction network, participate in the system to build protein score (median confidence) $>0.4$. The critical genes of upper respiratory tract infection were screened by Molecular Complex Detection (MCODS) v1.4.2.

\subsection{Gene Ontology Analysis and Pathway Analysis of Key Gene}

Key genes corresponding to the main chemical components of compound Honggencao were used for gene ontology analysis and pathway analysis, including biological process (BP), molecular function (MF) and cellular component (CC). Using R software "org. Hs. Eg. Db", "clusterProfiler", "enrichplot" and "ggplot2" package analysis [7], the adjusted p-value is less than 0.05 .

\section{Results}

\subsection{Chemical Constituents of a Compound}

The main chemical components of compound Honggencao were obtained from TCMSP, including a total of 69 parts as shown in Table 1. The corresponding target genes were a total of 1090, and 205 were left after the removal of the duplicates. Six thousand three hundred ninety-one target genes of upper respiratory tract infection were obtained from the GeneCards database. Finally, 186 target genes of compound Honggencao and upper respiratory tract infection were obtained, as shown in Figure 1.

\subsection{Construct the Network of Active Compounds of Compound Honggencao-Targets}

Based on the above data of active compounds of compound Honggencao and upper respiratory tract infection from GeneCards database, Cytoscape software was used in this study to construct a target network diagram (as shown in Figure 2) related to compound components of Honggencao and upper respiratory tract infection, with 256 nodes and 1079 edges (Figure 2).

\subsection{Construction of Protein Interaction Network}

A protein interaction network consisting of 186 nodes and 3515 edges was constructed in STING from the compound components corresponding to the targets related to upper respiratory tract infection (Figure 3). MCODSv1.4.2, a plug-in of Cytoscape, was used to analyse and obtain the critical gene network 
relationships of the main chemical components of compound Honggencao concerning upper respiratory tract infection targets, as shown in Figure 4. There are 56 nodes and 1228 edges.

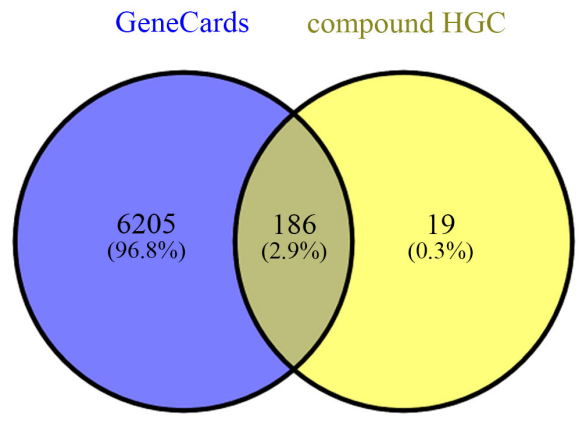

Figure 1. The targets of compound Honggencao concerning upper respiratory tract infection. (Blue circle shows there are 6391 target genes of upper respiratory tract infection were obtained from the GeneCards database. Yellow circle shows there are 205 genes in compound Honggencao. The intersection of two circles shows there are 186 targets in compound Honggencao are associated with treatment in upper respiratory tract infection.)

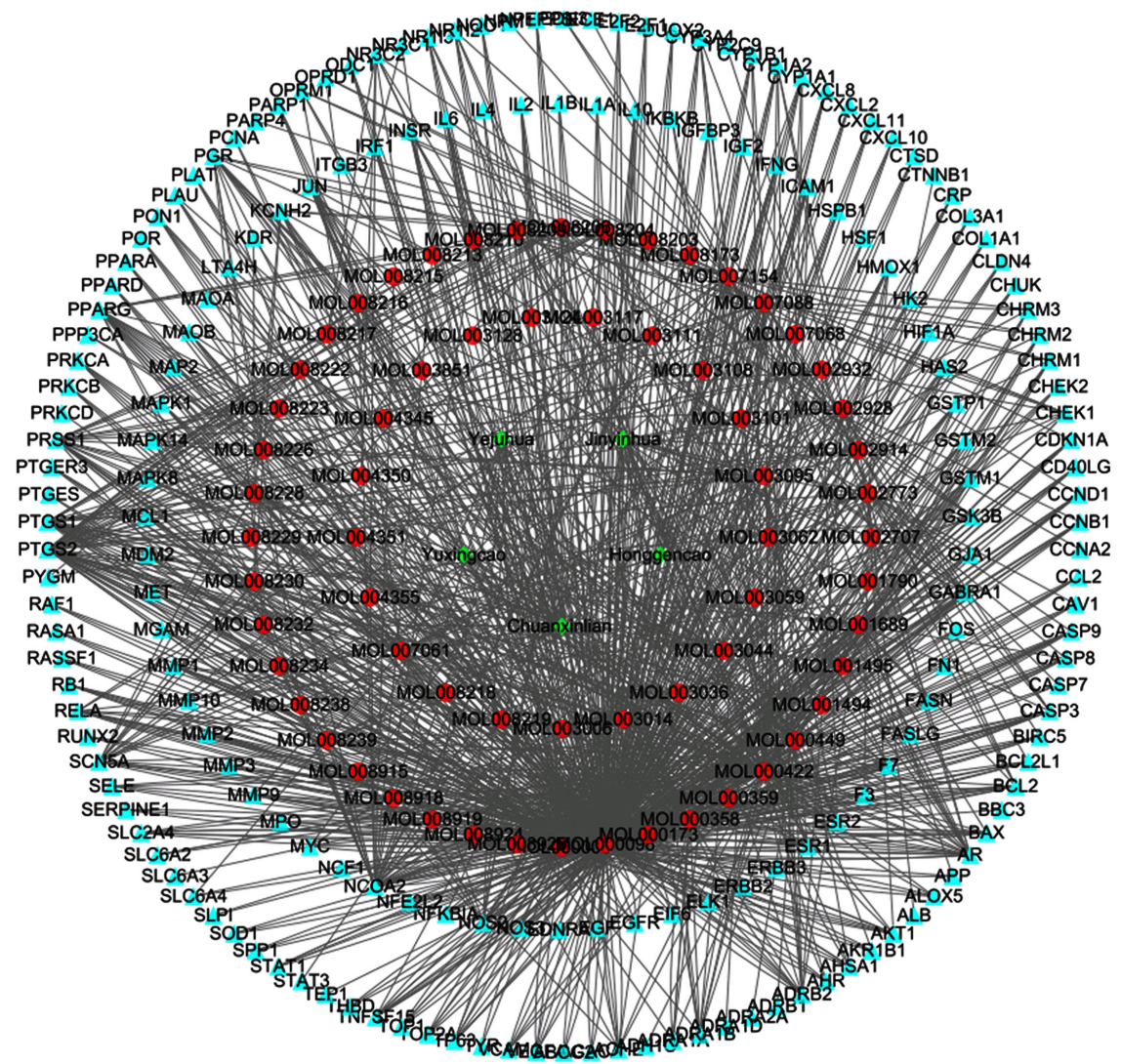

Figure 2. Target network of compound Honggencao of active components related to upper respiratory tract infection. (There are compounds in red, traditional Chinese medicine in green, targets in blue.) 


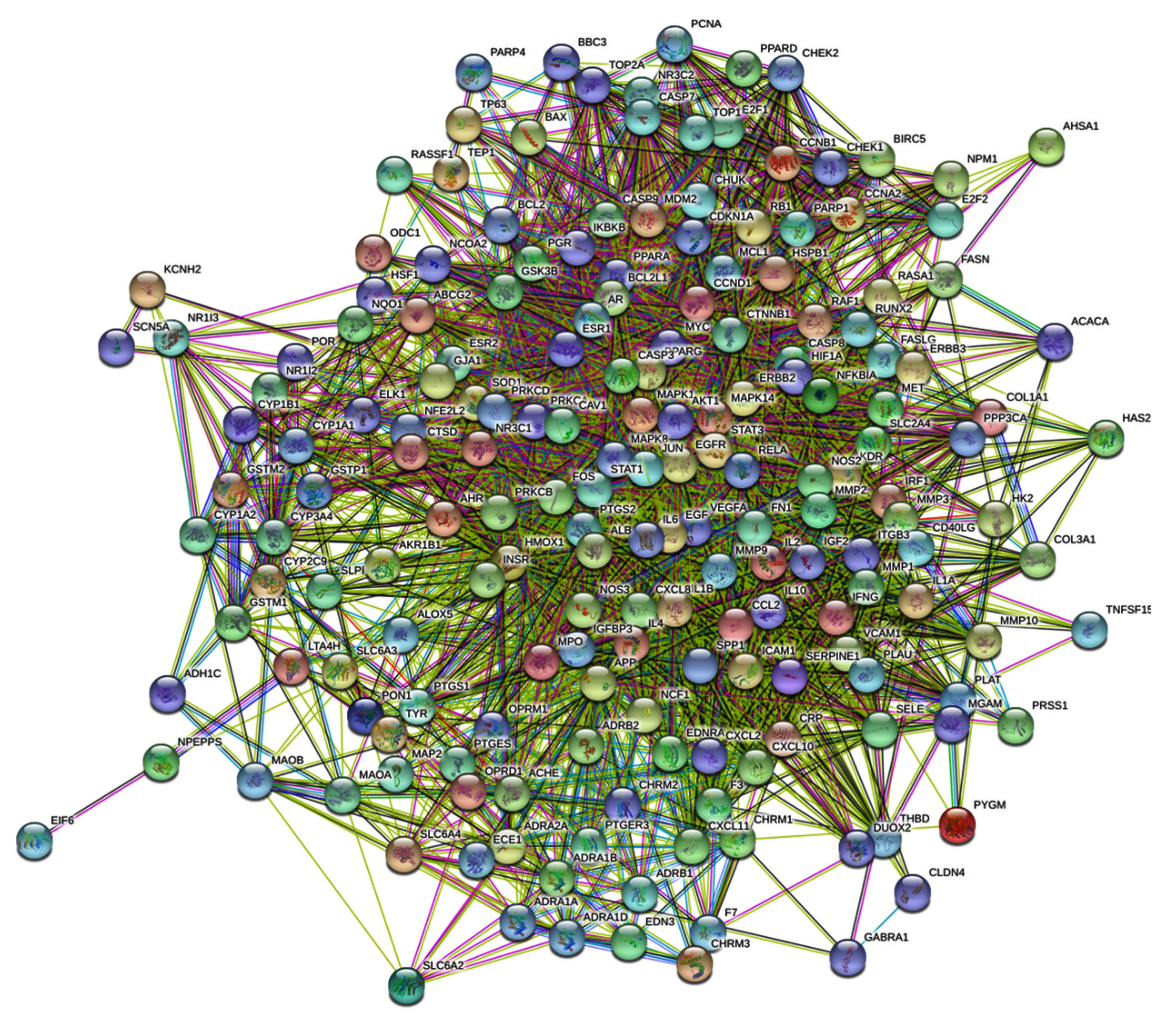

Figure 3. Interaction network relationship of potential action targets of compound Honggencao.

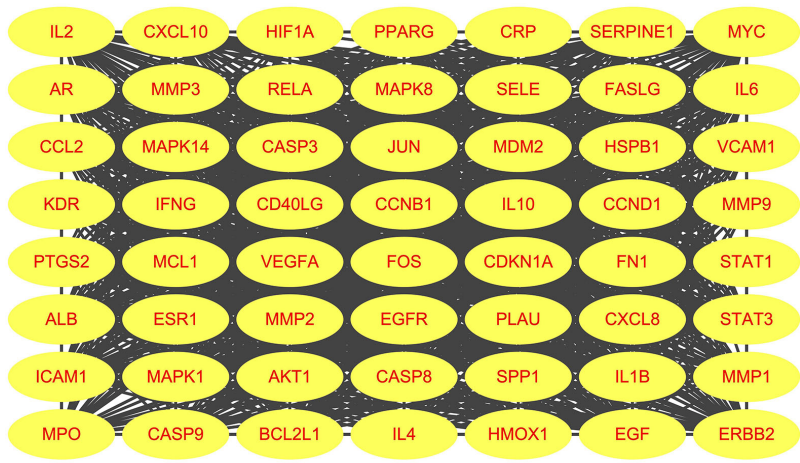

Figure 4. Key gene interaction network relationship of compound Honggencao of active components related to upper respiratory tract infection.

Table 1. Basic information of active compounds in compound Honggencao.

\begin{tabular}{|c|c|c|c|c|c|}
\hline NO. & Active compounds & MOL ID & Molecular weight & OB $(\%)$ & DL \\
\hline & Honggencao (Salvia prionit & & & & \\
\hline 1 & cryptotanshinone & MOL007088 & 296.39 & 52.34 & 0.4 \\
\hline 2 & Methylenetanshinquinone & MOL007061 & 278.32 & 37.07 & 0.36 \\
\hline 3 & Przewaquinone B & MOL007068 & 292.30 & 62.24 & 0.41 \\
\hline 4 & tanshinone iia & MOL007154 & 294.37 & 49.89 & 0.4 \\
\hline
\end{tabular}




\section{Continued}

Jinyinhua (Lonicerae Japonicae Flos)

\begin{tabular}{|c|c|c|c|c|c|}
\hline 5 & Mandenol & MOL007088 & 308.56 & 42 & 0.19 \\
\hline 6 & Ethyl linolenate & MOL001494 & 306.54 & 46.1 & 0.2 \\
\hline 7 & phytofluene & MOL001495 & 543.02 & 43.18 & 0.5 \\
\hline 8 & Eriodyctiol (flavanone) & MOL002707 & 288.27 & 41.35 & 0.24 \\
\hline 9 & $\begin{array}{l}\text { (-)-(3R,8S,9R,9aS,10aS)-9-ethenyl-8-(beta-D-glucopyranosyloxy)- } \\
\text { 2,3,9,9a,10,10a-hexahydro-5-oxo-5H,8H-pyrano[4,3-d] oxazolo[3,2-a] } \\
\text { pyridine-3-carboxylic acid_qt }\end{array}$ & MOL002914 & 281.29 & 87.47 & 0.23 \\
\hline 10 & secologanic dibutylacetal_qt & MOL003006 & 384.57 & 53.65 & 0.29 \\
\hline 11 & beta-carotene & MOL003014 & 536.96 & 37.18 & 0.58 \\
\hline 12 & ZINC03978781 & MOL002773 & 412.77 & 43.83 & 0.76 \\
\hline 13 & Chryseriol & MOL003036 & 300.28 & 35.85 & 0.27 \\
\hline 14 & kryptoxanthin & MOL003044 & 552.96 & 47.25 & 0.57 \\
\hline 15 & 4,5'-Retro-.beta.,.beta.-Carotene-3,3'-dione, 4',5'-didehydro- & MOL003059 & 562.90 & 31.22 & 0.55 \\
\hline 16 & 5-hydroxy-7-methoxy-2-(3,4,5-trimethoxyphenyl)chromone & MOL003062 & 358.37 & 51.96 & 0.41 \\
\hline 17 & 7-epi-Vogeloside & MOL003095 & 432.47 & 46.13 & 0.58 \\
\hline 18 & Caeruloside C & MOL003101 & 550.57 & 55.64 & 0.73 \\
\hline 19 & Centauroside_qt & MOL003108 & 434.48 & 55.79 & 0.5 \\
\hline 20 & Ioniceracetalides B_qt & MOL003111 & 314.37 & 61.19 & 0.19 \\
\hline 21 & XYLOSTOSIDINE & MOL003117 & 415.51 & 43.17 & 0.64 \\
\hline 22 & dinethylsecologanoside & MOL003124 & 434.44 & 48.46 & 0.48 \\
\hline 23 & beta-sitosterol & MOL003128 & 414.79 & 36.91 & 0.75 \\
\hline 24 & kaempferol & MOL000358 & 286.25 & 41.88 & 0.24 \\
\hline 25 & Stigmasterol & MOL000422 & 412.77 & 43.83 & 0.76 \\
\hline 26 & luteolin & MOL000449 & 286.25 & 36.16 & 0.25 \\
\hline 27 & quercetin & MOL000006 & 302.25 & 46.43 & 0.28 \\
\hline 28 & wogonin & MOL000098 & 284.28 & 30.68 & 0.23 \\
\hline & Chuanxinlian (Andrographis Herba) & & & & \\
\hline 29 & oroxylin a & MOL000173 & 284.28 & 41.37 & 0.23 \\
\hline 30 & Panicolin & MOL002928 & 314.31 & 76.26 & 0.29 \\
\hline 31 & 14-deoxy-11-oxo-andrographolide & MOL002932 & 348.48 & 57.06 & 0.34 \\
\hline 32 & Mono-O-methylwightin & MOL008203 & 358.37 & 103.11 & 0.4 \\
\hline 33 & Moslosooflavone & MOL008204 & 298.31 & 44.09 & 0.25 \\
\hline 34 & Deoxycamptothecine & MOL008206 & 332.38 & 50.01 & 0.77 \\
\hline 35 & Deoxyelephantopin & MOL008209 & 344.39 & 105.32 & 0.4 \\
\hline 36 & 14-deoxy-12-methoxyandrographolide & MOL008210 & 364.53 & 70.29 & 0.36 \\
\hline 37 & Paniculide B & MOL008213 & 280.35 & 52.27 & 0.21 \\
\hline 38 & Paniculide C & MOL008215 & 278.33 & 79.73 & 0.21 \\
\hline 39 & Paniculogenin & MOL008216 & 448.71 & 47.66 & 0.75 \\
\hline
\end{tabular}


J. H. Hu et al.

\section{Continued}

\begin{tabular}{|c|c|c|c|c|c|}
\hline 40 & 1-Monoolein & MOL008217 & 356.61 & 34.13 & 0.3 \\
\hline 41 & $\begin{array}{l}\text { 3-[2-[(1R,4aS,5R,8aS)-5,8a-dimethyl-2-methylene-5-methylol-decalin-1-yl] } \\
\text { ethyl]-5H-furan-2-one }\end{array}$ & MOL008218 & 318.50 & 51.78 & 0.28 \\
\hline 42 & andrographidine B_qt & MOL008219 & 330.31 & 72.72 & 0.33 \\
\hline 43 & andrographidine $\mathrm{C}$ & MOL008222 & 460.47 & 56.85 & 0.77 \\
\hline 44 & 14-deoxyandrographolide & MOL008223 & 334.50 & 56.3 & 0.31 \\
\hline 45 & Andrographin & MOL008226 & 328.34 & 37.57 & 0.33 \\
\hline 46 & Andrographin F & MOL008228 & 536.53 & 33.34 & 0.85 \\
\hline 47 & andrographidine $\mathrm{F}_{-} \mathrm{qt}$ & MOL008229 & 374.37 & 77.13 & 0.45 \\
\hline 48 & $\begin{array}{l}\text { (3Z,4S)-3-[2-[(1R,4aS,5R,6R,8aS)-6-hydroxy-5,8a-dimethyl-2-methylene-5- } \\
\text { methylol-decalin-1-yl]ethylidene]-4-hydroxy-tetrahydrofuran-2-one }\end{array}$ & MOL008230 & 350.50 & 46.96 & 0.36 \\
\hline 49 & andrographolide-19- $\beta$-D-glucoside_qt & MOL008232 & 350.50 & 53.44 & 0.35 \\
\hline 50 & andrographolide-19- $\beta$-D-glucoside_qt & MOL008234 & 318.50 & 63.54 & 0.28 \\
\hline \multirow[t]{2}{*}{51} & Quercetin tetramethyl $\left(3^{\prime}, 4^{\prime}, 5,7\right)$ ether & MOL008238 & 358.37 & 31.57 & 0.41 \\
\hline & Yuxingcao (Houttuyniae Herba) & & & & \\
\hline 52 & Isoramanone & MOL003851 & 348.53 & 39.97 & 0.51 \\
\hline 53 & kaempferol & MOL000422 & 286.25 & 41.88 & 0.24 \\
\hline 54 & 1-methyl-2-nonacosyl-4-quinolone & MOL004345 & 566.07 & 31.54 & 0.5 \\
\hline 55 & Ruvoside_qt & MOL004350 & 390.57 & 36.12 & 0.76 \\
\hline 56 & $\mathrm{C} 09747$ & MOL004351 & 296.35 & 37.28 & 0.25 \\
\hline 57 & Spinasterol & MOL004355 & 412.77 & 42.98 & 0.76 \\
\hline \multirow[t]{2}{*}{58} & quercetin & MOL000098 & 302.25 & 46.43 & 0.28 \\
\hline & Yejuhua (Chrysanthemi Indici Flos) & & & & \\
\hline 59 & acacetin & MOL001689 & 284.28 & 34.97 & 0.24 \\
\hline 60 & Linarin & MOL001790 & 592.6 & 39.84 & 0.71 \\
\hline 61 & beta-sitosterol & MOL000358 & 414.79 & 36.91 & 0.75 \\
\hline 62 & sitosterol & MOL000359 & 414.79 & 36.91 & 0.75 \\
\hline 63 & luteolin & MOL000006 & 286.25 & 36.16 & 0.25 \\
\hline 64 & daucosterol_qt & MOL008173 & 414.79 & 36.91 & 0.75 \\
\hline 65 & acacetin-7-O- $\beta$-D-galactopyranoside & MOL008915 & 446.44 & 50.19 & 0.77 \\
\hline 66 & Arteglasin A & MOL008918 & 304.37 & 52.45 & 0.33 \\
\hline 67 & $\begin{array}{l}(2 \mathrm{~S}, 6 \mathrm{~S}, 7 \mathrm{aR})-2-[(1 \mathrm{E}, 3 \mathrm{E}, 5 \mathrm{E}, 7 \mathrm{E}, 9 \mathrm{E}, 11 \mathrm{E}, 13 \mathrm{E}, 15 \mathrm{E})-16-[(4 \mathrm{~S})-4 \text {-hydroxy-2,6,6- } \\
\text { trimethyl-1-cyclohexenyl]-1,5,10,14-tetramethylhexadeca-1,3,5,7,9,11,13,15- } \\
\text { octaenyl]-4,4,7a-trimethyl-2,5,6,7-tetrahydrobenzofuran-6-ol }\end{array}$ & MOL008919 & 584.96 & 59.52 & 0.55 \\
\hline 68 & $\begin{array}{l}\text { Azuleno(4,5-b)furan-2(3H)-one, } \\
\text { 4-(acetyloxy)-3a,4,5,6,6a,7,9a,9b-octahydro-6-hydroxy-6,9-dimethyl-3- } \\
\text { methylene-, (3aR-(3aalpha,4alpha,6alpha,6aalpha,9aalpha,9bbeta))- }\end{array}$ & MOL008924 & 306.39 & 68.44 & 0.27 \\
\hline 69 & $\begin{array}{l}\text { (3aR,4S,6R,6aR,9aR,9bR)-4,6-dihydroxy-6,9-dimethyl-3-methylene- } \\
\text { 4,5,6a,7,9a,9b-hexahydro-3aH-azuleno[5,4-d]furan-2-one }\end{array}$ & MOL008925 & 264.35 & 40.08 & 0.19 \\
\hline 70 & quercetin & MOL000098 & 302.25 & 46.43 & 0.28 \\
\hline
\end{tabular}




\subsection{GO Analysis and Pathway Analysis of Essential Genes}

Through the study of the critical gene ontology, there were 2567 GO entries, among which BP included 2273 such as response to lipopolysaccharide, response to molecule of bacterial origin and response to oxygen levels. CC contains 103 items including Clinique, clinique microdomain and clinique region. There were $191 \mathrm{MF}$, including nuclear receptor activity, transcription factor activity, direct ligand regulated sequence-specific DNA binding and cytokine receptor binding. The results are shown in Figure 5 for the top 10 GO analysis. There were 166 pathways involved in pathway analysis, including AGE-RAGE signaling pathway in diabetic complications, PFluid shear stress and atherosclerosis and Human cytomegalovirus infection. The results of GO analysis and KEGG analysis displayed in Figure 5.

\section{Discussion}

Network pharmacology, as a method to investigate the network relationship among drugs, diseases and targets, has been widely used in pharmacology research and is helpful to find the target and molecular mechanism of drug action quickly. We have reported on the molecular mechanism of chemical components of Honggencao based on network pharmacology and found that the pathways of Honggencao were related to Staphylococcus aureus infection and hepatitis B infectious diseases [6]. Du et al. reported that Jinyinghua exhibited the characteristics of multi-molecule, multi-target and multi-pathway in the anti-inflammatory mechanism of its active ingredient based on network pharmacology [8]. Wang et al. also investigated the mechanism of action of Yuxingcao in the treatment of bronchitis based on network pharmacology and found 12 active components and 42 bronchitis targets [9]. In this study, we will further systematically explore the active components and targets of compound Honggencao on upper respiratory tract infection using network pharmacology, and reveal the interaction between the upper respiratory tract infection and the active components of compound Honggencao on upper respiratory tract infection.

Liu Wei found that the serum level of IL-17 in children with recurrent upper respiratory tract infection was significantly higher than that of healthy children. Cox statistical analysis showed that IL-17 was an independent factor in the occurrence of children with upper respiratory tract infection [10]. Our results found that the active component pathway of compound Honggencao included IL-17 signaling pathway. Therefore, combined with the characteristics of IL-17 expression level in the mentioned clinical reports, certain theoretical basis can be provided for the treatment of upper respiratory tract infection by compound Honggencao. Ma et al. reported that in patients treated with Yuxingcao combined with amoxicillin for upper respiratory tract infection, the tumour necrosis factor-a level was significantly lower than that treated with amoxicillin [11], which was consistent with the pathway of action of the active components of compound Honggencao including tumour necrosis factor signaling pathway in 


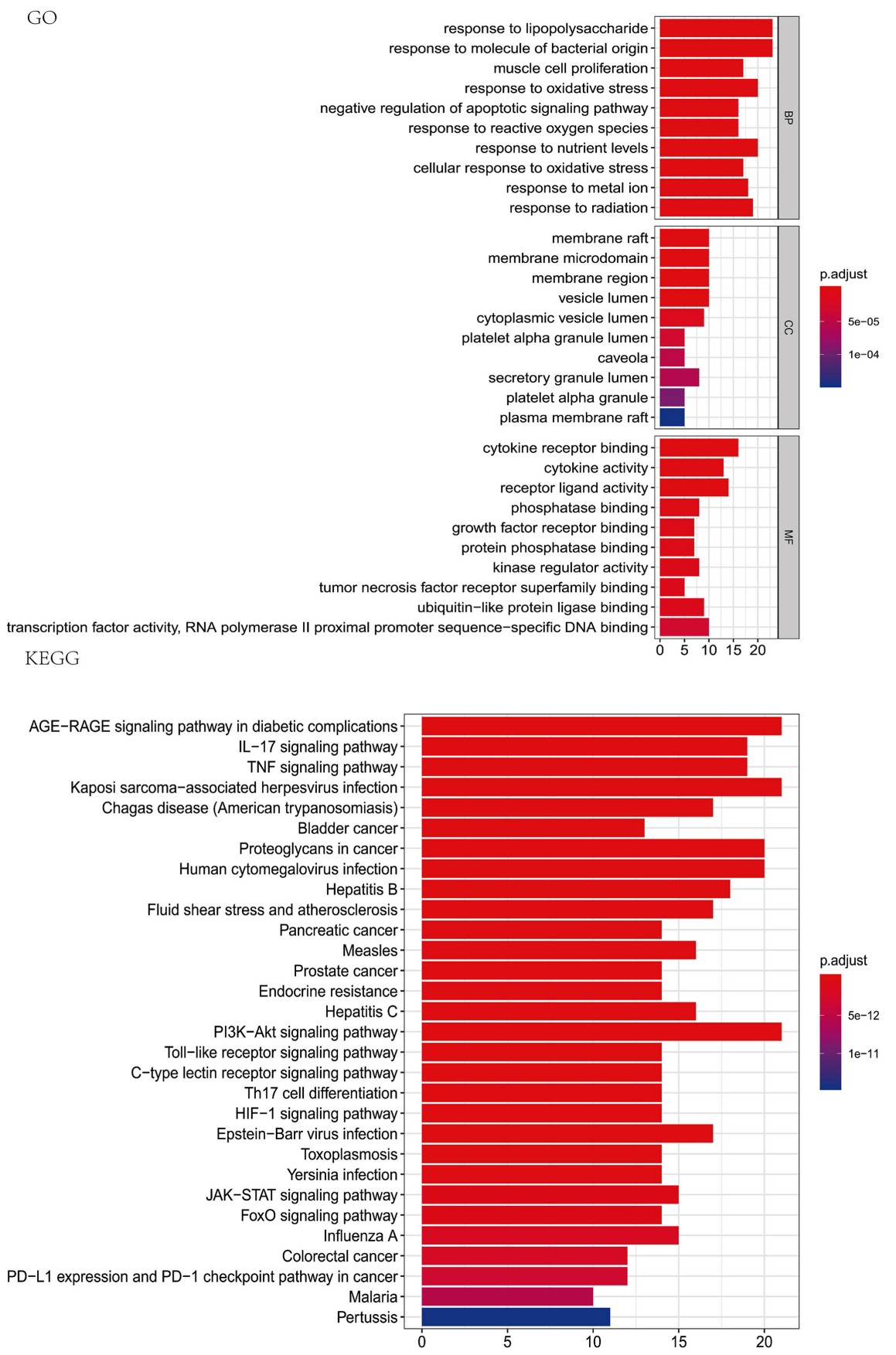

Figure 5. The top $10 \mathrm{GO}$ analysis and top 20 KEGG pathways of compound Honggencao.

this study. The action pathway of compound Honggencao also included the human cytomegalovirus infection pathway, so in the infantile idiopathic thrombocytopenic purpura cytomegalovirus infection, its precursor infection was mainly the upper respiratory tract infection [12], which provided a certain theoretical reference for the use of compound red root in the prevention and treatment of the infection with the help of network pharmacology.

In this study, although it found that compound Honggencao had the characteristics of multiple components and multiple targets, and explained its related 
signal channels in upper respiratory tract infection, the complex network relationship between its drugs and the disease still need to be further verified by experiment and clinic.

\section{Conclusion}

Based on the method of network pharmacology, this study explored the potential active components, targets and signaling pathways of compound Honggencao in the treatment of upper respiratory tract infection, providing a certain theoretical basis for the research on the pharmacological direction of molecular signaling pathways and providing a certain theoretical basis for clinical use.

\section{Acknowledgements}

This study was funded by the Study and Development Fund for Sciences and Technology in Chengde City (No. 201701A086 and No. 201701A087).

\section{Consent for Publication}

All of the authors consent to the publication of this study in the Journal of Chinese Medicine.

\section{Conflicts of Interest}

The authors declare no conflicts of interest regarding the publication of this paper.

\section{References}

[1] Rong, P., Ma, R., Liu, Q.H., Yan, H.H., Hu, S.Y., Li, X.M., Zhang, X.L. and Zheng, W.K. (2017) A Commentary of Literature Research of Traditional Chinese Medicine for Acute Upper Respiratory Tract Infection in Children. China Journal of Chinese Materia Medica, 42, 1455-1466.

[2] Zhong, C.L., Shen, W., Cai, Q.H. and Hu, S.Y. (2017) Research Progress on Chinese Patent Medicine in Treatment of Acute Upper Respiratory Tract Infection in Children. Drugs \& Clinic, 32, 1600-1604.

[3] Zeng, R., Yan, M. and Liu, J.C. (2006) Study on the Extraction Conditions of Compound Honggencao by Orthogonal Experiment. Hunan Journal of Traditional Chinese Medicine, 22, 81-83.

[4] Zeng, R., Yan, M. and Liu, J.C. (2006) HPLC Determination of Andrographolide and Dehydrated Andrographolide in Compound Honggencao Capsules. Journal of Hunan College of Traditional Chinese Medicine, 26, 25-26+28.

[5] Ru, J.L., Li, P., Wang, J., Zhou, W., Li, B., Huang, C., Li, P.D., Guo, Z., Tao, W.Y., Yang, Y., Xu, X., Li, Y., Wang, Y.H. and Yang, L. (2014) TCMSP: A Database of Systems Pharmacology for Drug Discovery from Herbal Medicines. Journal of Cheminformatics, 6, 13. https://doi.org/10.1186/1758-2946-6-13

[6] Hu, J.H., Yao Y.H., Wang, Y., Zhao, J.Y. and Jiang Y.P. (2019) Investigate of Molecular Mechanism of Salvia prionitis Based on Network Pharmacology. Modern Chinese Medicine, 21, 1505-1511.

[7] Yu, G.C., Wang, L.G., Han, Y.Y. and He, Q.Y. (2012) clusterProfiler: An R Package 
for Comparing Biological Themes among Gene Clusters. OMICS: A Journal of Integrative Biology, 16, 284-287. https://doi.org/10.1089/omi.2011.0118

[8] Du, Y.Q., Duan, Z.K., Dong, S.H., Zhao, P. and Song, S.J. (2019) Network Pharmacology Analysis of the Anti-Inflammatory Pharmacological Mechanisms of Active Ingredients from Lonicera japonica. Chinese Journal of Medicinal Chemistry, 29, 96-102.

[9] Wang, R., Fan, Q.M., Li, W.J., Chen, S.Y., Liu, H., He, P., Li, H.Y., Liu, W.L. and He, F.Y. (2019) Study on Mechanism of Houttuyniae Herba for Treatment of Bonchitis Based on Network Pharmacology. Chinese Journal of Information on Traditional Chinese Medicine, 26, 91-95.

[10] Liu, W. (2018) Levels and Clinical Significances of Serum 25-Hydroxyvitamin D3, IL-10 and IL-17 in Children with Recurrent Upper Respiratory Infection. China Medical Herald, 15, 80-83.

[11] Ma, R., Wang, R.T. and Di, Z.H. (2018) Clinical Study of Yuxingcao Injection Combined with Amoxicillin in Treatment of Upper Respiratory Tract Infection. Drugs \& Clinic, 33, 1950-1953.

[12] Wang, X. and Qiao, L.J. (2011) Clinical Observation for Cytomegalovirus Infection of Infant Idiopathic Thrombocytopenic Purpura. Journal of China Pediatric Blood and Cancer, 16, 268-271. 